\title{
Unveiling the mystery of "missing" emergencies in the era of COVID-19 pandemic: a generalized phenomenon with unclarified implications
}

\author{
Konstantinos Tsioufis ${ }^{1} \mathbb{D} \cdot$ Christina Chrysohoou $^{1} \cdot$ Ioannis Leontsinis ${ }^{1} \cdot$ Dimitrios Tousoulis $^{1}$
}

Received: 26 June 2020 / Accepted: 29 June 2020 / Published online: 23 July 2020

(c) Springer-Verlag GmbH Germany, part of Springer Nature 2020

\section{Sirs:}

A significant reduction concerning the hospital admissions for acute coronary syndromes (ACS), stroke, and cardiac catheterization rates has been documented both in Europe and the U.S. during the COVID-19 pandemic [1-3]. In like manner, our study revealed that all-cause visits at the Emergency Cardiology Department (ECD) and particularly the STEMI cases appeared to be greatly affected over the same period [4]. Interestingly, both the daily patients' visits in the ECD and myocardial infarction cases were inversely related with the COVID-19 spread trends in Greece [4].

It is difficult to explain the phenomenon of the "missing" emergency hospital visits. The results of our study refer to a tertiary Heart center which is not a COVID-19 dedicated hub. This may have contributed to these "missing" visits as some cases could have been visiting other healthcare facilities, misinterpreting heart-related symptoms as a probable infection. The centrally issued recommendation, however, prompted patients to reach COVID-19 dedicated centers only in case of high suspicion of a chest infection. For this reason, we could presume that cases with predominant heartrelated symptoms should not have been directed to other healthcare units. On the other hand, the fear of acquiring COVID-19 infection was recognized as the main reason to keep patients away from hospitals [3]. It is reasonable to hypothesize that this could have affected the visiting rates in COVID-19 dedicated hospitals equally, if not more. However, there are no available data yet to support such a hypothesis.

Data regarding a reduced mean left-ventricular ejection fraction (LVEF) among MI cases comprises another

Konstantinos Tsioufis

ktsioufis@hippocratio.gr

1 First Cardiology Clinic, Medical School, National and Kapodistrian University of Athens, Hippokration Hospital, 114 Vass Sofias Ave., 11527 Athens, Greece important finding about the ACS-related morbidity during the pandemic. In our study, before and post-COVID-19 pandemic, there was a significant decline of the observed mean LVEF among the STEMI cases between January 2020 and April 2020; from 47.8 to $37 \%$ accordingly $(p=0.031)$. This observation could be related with delays in presentation or initiation of treatment; however, other factors, such as renal function, diabetes mellitus, and excessive atherosclerosis, could also play an important role [3]. On the other hand, one could speculate that the reduced LVEF reflects missing cases with milder symptoms. Intriguingly, Hammad et al. showed that the culprit lesion was more likely to be localized in the left anterior descending (LAD) artery among the post-COVID-19 pandemic STEMI cases [3]. LAD-related infarctions are generally accompanied by lower LVEF on arrival [5]. However, it should be acknowledged that in several cases, especially in older patients, the estimation of EF is not enough for the evaluation of ventricular function, as ventricular stiffness and deformation of longitudinal strain rate are related to clinical outcome, while the improvement in these two parameters reflects pressure overload recovery.

Cardiovascular disease remains the leading cause of premature death and disability among the western populations with increasing rates globally. Current estimations suggest that COVID-19 epidemic will lead to long-lasting unfavorable economic effects, not only to individuals, but also on health systems increasing future demands for healthcare services, since all postponed elective and semi-elective procedures will accumulate, blocking hospitals capacity. Based on the current data, we aim to conduct a prospective study to evaluate the short- and longer-term implications of the pandemic.

Being optimistic, this pandemic can be viewed as an opportunity to enhance the promotion of e-visits, patient's tele-monitoring, initiation of e-health programs, and above all to raise public awareness so as to be prepared for future challenges. 
Funding None.

\section{Compliance with ethical standards}

Conflict of interest The authors declare that they have no conflict of interest.

\section{Reference}

1. Gupta T et al (2020) Impact of the COVID-19 pandemic on interventional cardiology fellowship training in the New York metropolitan area: a perspective from the United States epicenter. Catheter Cardiovasc Interv. https://doi.org/10.1002/ccd.28977
2. de Havenon A et al (2020) A rapid decrease in stroke, acute coronary syndrome, and corresponding interventions at 65 united states hospitals following emergence of COVID-19. Cardiovasc Med. https://doi.org/10.1101/2020.05.07.20083386

3. Hammad TA et al (2020) Impact of COVID-19 pandemic on STelevation myocardial infarction in a non-COVID-19 epicenter. Catheter Cardiovasc Interv. https://doi.org/10.1002/ccd.28997

4. Tsioufis $\mathrm{K}$ et al (2020) The mystery of 'missing' visits in an emergency cardiology department, in the era of COVID-19; a time-series analysis in a tertiary Greek General Hospital. Clin Res Cardiol. https://doi.org/10.1007/s00392-020-01682-1

5. Chen $S$ et al (2019) Renal denervation for mild-moderate treatment-resistant hypertension: a timely intervention? Herz 44(5):412-418. https://doi.org/10.1007/s00059-017-4664-0 